\title{
eJRIEPS
}

Ejournal de la recherche sur l'intervention en éducation physique et sport

$36 \mid 2015$

Varia

\section{Analyse didactique clinique des savoirs enseignés par un entraineur en basket-ball}

Clinical analysis of didactic knowledge taught by a basketball coach

\section{Romain Chonaki et Denis Loizon}

\section{(2) OpenEdition}

\section{Journals}

Édition électronique

URL : http://journals.openedition.org/ejrieps/1446

DOI : 10.4000/ejrieps. 1446

ISSN : 2105-0821

Éditeur

ELLIADD

\section{Référence électronique}

Romain Chonaki et Denis Loizon, « Analyse didactique clinique des savoirs enseignés par un entraineur en basket-ball », eJRIEPS [En ligne], 36 | 2015, mis en ligne le 01 juillet 2015, consulté le 08 janvier 2020. URL : http://journals.openedition.org/ejrieps/1446; DOI : 10.4000/ejrieps. 1446

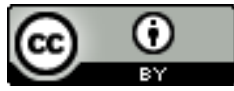

La revue eJRIEPS est mise à disposition selon les termes de la Creative Commons Attribution 4.0 International License. 
eJRIEPS 36 juillet 2015

\section{Analyse didactique clinique des savoirs enseignés \\ par un entraineur en basket-ball.}

Romain Chonaki et Denis Loizon

Université de Bourgogne, Dijon, France.

\section{Résumé}

Cette recherche s'inscrit dans le cadre scientifique de la didactique clinique de l'Education Physique et Sportive (EPS). Sa caractéristique repose sur la prise en compte du sujet enseignant, de son histoire et de ses effets sur sa pratique d'enseignement. Cet article analyse la transmission du savoir par un entraîneur de basket-ball au regard de son expérience dans l'activité. L'étude de cas d'un professionnel de métier, permet de rendre compte d'une double nature de savoirs enseignés : des savoirs techniques, prioritaires et diversifiés, et des savoirs tactiques, globalement rattachés à la gestion du jeu. Elle met également en perspective l'existence d'un décalage entre les savoirs enseignés réellement par l'entraîneur et les savoirs qu'il pense enseigner (les savoirs virtuels), démontrant la trace d'enseignements qui ne faisaient initialement pas partie des objectifs de l'enseignement, ou enseignements incidents.

Mots clés : basket-ball, conversion didactique, didactique clinique, savoirs.

\section{Introduction}

Cet article a pour but de rendre compte de la transmission des savoirs par un entraîneur de basket-ball professionnel à un groupe de jeunes basketteurs de haut niveau. La diffusion de savoirs enseignés précis à partir de processus pédagogiques mis en place sur des séances par l'entraîneur constitue le cœur de cette étude.

Dans une approche clinique de la didactique, nous nous centrons sur le sujet entraîneur et son histoire personnelle (Terrisse, Carnus, 2009) mettant ainsi en évidence son " déjàlà ", notion devenue incontournable dans les recherches en didactique clinique (Carnus, 2003). Ce " déjà-là » permet de définir d'une part la conception que se fait l'entraîneur de l'activité qu'il enseigne et celle qu'il se fait de sa transmission (Terrisse, 2009) afin d'en " comprendre l'activité didactique » (Loizon, Carnus, 2011). La prise en compte du vécu et de l'expérience de l'activité du sujet, déterminés à partir de plusieurs entretiens d'accès au 


\section{eJRIEPS 36 juillet 2015}

déjà-là, permet au chercheur de comprendre leur pertinence dans le processus de transmission et d'appropriation du savoir.

Dans cette perspective, le rapport au savoir de l'enseignant demeure central et conduit à un objet de recherche précis: il s'agit de déterminer le poids de la "conversion didactique » (Buznic-Bourgeacq, 2005) de l'entraîneur dans l'activité basket-ball. Nous cherchons d'une part à identifier la nature des savoirs enseignés, à partir de l'expérience de l'entraîneur, et d'autre part, à déterminer s'il existe une trace de savoirs enseignés incidents inscrits dans un écart entre les savoirs que l'entraîneur souhaite enseigner (ses intentions didactiques) et ceux qu'il a véritablement enseignés (les savoirs appris par les joueurs).

Nous situons notre recherche dans le prolongement des études menées par l'équipe EDiC (Université de Toulouse le Mirail) et plus précisément dans la continuité des travaux de JP. Sauvegrain (2001), qui a approfondi la question des relations entre pratiques enseignantes et apprentissages réels des élèves dans l'activité lutte.

\section{Le cadre théorique}

Nos options de recherche se situent d'abord dans le champ de la didactique de l'EPS, dont les notions de savoir, d'objets de savoir, de rapport au savoir et de transposition didactique demeurent centrales. Cette orientation scientifique qui complète le champ de la didactique par celui de la clinique d'inspiration psychanalytique permet de nous appuyer aussi bien sur des concepts faisant référence à la didactique, que sur des éléments propres à la psychanalyse afin de mieux comprendre et d'analyser les enjeux de savoirs dans le processus d'enseignement de l'entraîneur.

2.1. Une question de savoir

La large palette des définitions données pour la notion de savoir par certains théoriciens tend à montrer un déficit de consensus autour de cette acception: entre les “ connaissances déclaratives" (Delignières, Duret, 2004), les "science, information, compétence, cognition, croyance, contenu, représentation, processus... » (Raynal, Rieunier, 1997), les « compétence et interdisciplinarité » (Astolfi, 2008), les définitions du savoir sont vastes. Néanmoins, nous nous rapprochons de la définition de J. Beillerot (1989, p.6) pour qui le savoir représente "ce qui, pour le sujet, est acquis, construit, élaboré par l'étude ou l'expérience ». 


\section{eJRIEPS 36 juillet 2015}

Dans notre objectif de théorisation du sujet entraîneur, que nous sommes amenés à construire à partir de cette approche didactique clinique, il convient de préciser encore deux formes de savoir :

- Le savoir " savant » (Joshua, 1996), perçu comme un amas de connaissances empilées, rigoureusement triées et susceptibles d'être réinvesties très vite, sous la base de procédés déclaratifs immédiats ; c'est celui qui est construit a priori par l'entraîneur.

- Le savoir « expert » (Joshua, 1996), que D. Bouthier et A. Durey (1994) nomment savoir "technologique ", déterminé comme la conséquence d'évènements vécus, gravés à jamais dans notre moi (au sens psychanalytique du terme), qui apparaît plus comme le produit abstrait d'une transformation cognitive. "Le savoir est infini, multiforme, sans contour discernable. S'il est savoir pour la pensée, il devient savoir-faire pour l'action » (Malglaive, 1987, p. 285). Ce savoir-là est à la base du savoir "savant » que s'est construit l'entraîneur dans son histoire de pratiquant.

Dans les activités physiques et sportives (APSA), les savoirs sont issus des « pratiques sociales de référence ", mais peuvent évoluer au gré du contexte sociétal. "La référence dans les APSA est surtout le savoir d'expert, celui qu'utilisent les champions, même s'il ne peut être transmis tel quel à l'école » (Terrisse, 1999, p. 16). La complexité vient donc de la capacité de l'entraîneur à transmettre un savoir adapté à ses joueurs.

Plus spécifiquement, J-F. Gréhaigne (1999) évoque dans l'activité football des savoirs bien précis : d'abord, il évoque des "savoirs tactiques ", qui représentent pour A. Terrisse (2000, p. 8) le «savoir pour la pratique ", dont les règles d'action en sont l'un des supports privilégiés, règles d'action qu'il définit comme étant « les conditions à respecter et les éléments à prendre en compte pour que l'action soit efficace ». C'est donc à partir de celles-ci que l'apprenant va se construire un répertoire de savoirs tactiques sur l'activité. D. Loizon (2008) parle plutôt d'un savoir «stratégique " assez proche, qui " peut être assimilé quant à lui à un savoir procédural dans la mesure où il donne lieu à des énoncés de type "opératif » " (Barbier, 1996, p.11). Ensuite, il définit des «savoirs en action ", c'est-à-dire des "savoirs sur le jeu et des savoirs en jeu [...] en synergie avec les « compétences motrices » en vue d'être efficace sur le terrain » (p. 2). Ce sont donc à partir de ces savoirs que le joueur pourra se mouvoir dans la pratique du football, en interaction avec les nombreuses contraintes didactiques auxquelles il sera confronté. En complément, J-F. Gréhaigne et A. Fernandez (1999) définissent également des « savoirs de gestion ", constituant des savoirs plus précis qui participent à l'élaboration du jeu et permettent une forme « d'auto-management d'équipe ». 


\section{eJRIEPS 36 juillet 2015}

Dans l'optique de développer et d'analyser les savoirs en basket-ball, nous nous appuierons sur les études didactiques de J-F. Gréhaigne afin de distinguer trois types de savoirs. Plutôt que d'utiliser le terme «savoirs en action », nous préférons parler de « savoirs techniques» définis par D. Loizon (2008, p. 65), «comme l'ensemble des techniques qui relèvent de l'activité judo, techniques codifiées au fil du temps par les experts ». Cela nous permet de ne pas confondre certains aspects techniques et tactiques qui, pour nous, peuvent parfois être autant de savoirs en action et ainsi scinder véritablement des «savoirs techniques » et des «savoirs tactiques ». A ces deux types de savoirs, nous rajoutons un troisième type, les « savoirs méthodologiques », qui sont bien spécifiques au poste des deux joueurs qui serviront de support à notre recherche.

\subsection{Une question de rapport au savoir}

Le rapport au savoir, central dans le processus d'enseignement, est lui aussi fortement mobilisé dans les recherches en didactique clinique. Le chercheur va en effet essayer de déterminer «le processus par lequel un élève se transforme » (Terrisse, Carnus, 2009), à travers les situations que l'enseignant met en place.

« L'intérêt de l'outil « rapport au savoir » est d'analyser les transformations qu'induit l'apprentissage en terme de transformations motrices pour l'élève et de conception de l'enseignement pour le professeur » (Terrisse, Carnus, 2009, p.23). Le rapport au savoir de l'entraîneur « n'est accessible que par le biais de l'analyse des gestes professionnels » (Garnier, 2008). De plus, D. Loizon et al. (2008) ou encore P. Buznic-Bourgeacq et al. (2010, p.13) ont montré « le poids de l'expérience personnelle du professeur d'EPS dans l'activité enseignée sur ses pratiques d'enseignement ». Le contenu d'enseignement est beaucoup plus ouvert, dynamique, complexe et formulé plus précisément, en comparaison à un enseignant qui n'a que peu de vécu dans l'activité enseignée.

L'analyse du rapport au savoir de l'entraîneur devrait permettre de déterminer les véritables objets de savoir enseignés, qui seront le fruit de ce qu'il va chercher à transmettre à ses joueurs.

\subsection{Une question de transposition didactique}

D'abord envisagé par M. Verret (1975) dans le cadre de la sociologie de l'Education, la notion de «transposition didactique », revisitée depuis par Y. Chevallard (1985), rend compte de la transformation du savoir au cours du processus d'apprentissage et définit le chemin qui caractérise le passage du savoir savant au savoir enseigné (Chevallard, 1991). Pour lui, le « savoir savant » est un savoir socialement et institutionnellement avéré : « Le 


\section{eJRIEPS 36 juillet 2015}

titre de savant n'appartient jamais intrinsèquement à un savoir. II est accordé par la culture et il peut se perdre » (ibid, p.11).

Dans le cadre de l'enseignement des APSA, ces savoirs savants représentent des savoirs sur la pratique et ne sont pas toujours les objets de savoirs enseignés. Transposer didactiquement une forme de savoir savant, c'est donc construire un savoir théorique afin de permettre à une pratique sociale de référence de devenir une pratique sportive, dans laquelle on peut construire des situations d'enseignement. Ce savoir théorique, qui deviendra alors un savoir enseigné, reste pour l'entraîneur « à la fois une conception, souvent marquée du sceau de sa propre expérience, et une façon de l'enseigner, plutôt dépendante de l'idée qu'il se fait de son rôle d'enseignant » (Terrisse, 1999, p. 22).

Afin de construire un ensemble de données complet pour cette étude, nous avons donc choisi de retenir trois catégories de savoirs qui peuvent être enseignés en basket-ball : les savoirs techniques, les savoirs tactiques et les savoirs méthodologiques.

\subsection{Une question d'enseignements « incidents »}

Dans l'activité karaté, Heuser, Terrisse et Carnus (2005, p.71) ont montré qu'il existe un écart entre les savoirs enseignés et les savoirs appris : le savoir peut « découler d'un acte d'enseignement mais être aussi appris au cours même de l'épreuve, par l'épreuve ou encore être issu d'un savoir déjà-là exploité au cours de l'épreuve. Autrement dit, si le savoir utilisé nous renseigne sur le savoir appris, ce dernier n’a pas toujours été enseigné ».

Dans cette optique, puisqu'un apprenant est capable d'acquérir des savoirs « inventés », en utilisant « un savoir qui n'a pas été enseigné » (idid), nous faisons l'hypothèse qu'un savoir peut également être enseigné de manière involontaire.

En d'autres termes, nous pouvons envisager l'existence d'enseignements incidents, c'està-dire la présence d'une transmission involontaire de savoirs et donc d'un écart entre les savoirs réellement enseignés par l'entraîneur et les savoirs qu'il pense enseigner que l'on qualifiera de « savoirs virtuels».

2.5. Une étude de cas comme base d'étude

L'étude de cas s'inscrit ici dans une logique qualitative et une méthodologie adaptée à la didactique clinique. Elle permet de décrire, d'analyser et de comprendre les déterminants d'une situation d'enseignement en prenant en compte les rapports qui existent entre les sujets et l'activité. L'étude de cas peut se définir comme " la compréhension de dynamiques présentes au sein d'un environnement unique. Elle se limite à un domaine d'investigation bien spécifique et permet le recueil de données diverses » (Yin, 1984, 


\section{eJRIEPS 36 juillet 2015}

p.27). II s'agit donc d'une stratégie de recherche visant à faire ressortir les singularités des sujets à partir d'une logique qualitative.

Pour notre recherche, nous avons choisi comme cas un entraîneur de basket-ball professionnel et réputé pour ses qualités de formateur que nous nommerons Pierre. II est âgé de 26 ans, titulaire du Brevet d'Etat $1^{\text {er }}$ degré. II enseigne le basket-ball depuis l'âge de douze ans mais ne pratique plus pour raison de santé. En constante réflexion sur sa pratique, il n'hésite pas à tester et à construire des situations d'enseignement novatrices, dans l'optique de créer de nouvelles conduites motrices et de développer de nouveaux savoirs. Son investissement va bien au-delà du club dans lequel il est salarié, puisqu'il s'investit également au sein du comité départemental et de la ligue régionale.

\section{Question de recherche}

Notre étude repose sur l'analyse des savoirs enseignés en basket-ball par un entraîneur face à un groupe de joueurs de la catégorie U15 en basket-ball (ou under 15) national, c'est-à-dire à des joueurs de moins de quinze ans qui évoluent dans un championnat de niveau national.

Dans cette optique, le poids de la transmission de savoirs personnels acquis par l'expérience ou « conversion didactique » (Buznic-Bourgeacq, 2005) et son impact sur les apprentissages des joueurs sont au cœur de notre étude, puisqu'en s'appuyant sur l'expérience de l'entraîneur, nous cherchons à déterminer d'une part la nature des savoirs enseignés, et d'autre part, l'existence éventuelle d'une trace de savoirs enseignés incidents, c'est-à-dire d'un décalage entre les savoirs que l'entraîneur souhaite enseigner et ceux qu'il enseigne véritablement.

\section{Le cadre méthodologique}

4.1. Les trois temps de la méthodologie en didactique clinique

Cette méthodologie s'appuient sur une «théorie du sujet» (Terrisse, 2009). Ils représentent la structuration d'une méthodologie de type « clinique ».

\subsubsection{Le déjà-là}

Le premier temps de la recherche, celui d'avant l'épreuve, consiste à déterminer ce qui va orienter les intentions de Pierre. Pour cela, nous allons essayer de mettre en évidence son « déjà-là ». Celui-ci correspond à un ensemble très composite issu de l'histoire du sujet. Accéder à ce « déjà-là » et à ces savoirs d'expériences, préfigure la nature du savoir à enseigner, de son mode de transmission et des conditions de son appropriation. Des 


\section{eJRIEPS 36 juillet 2015}

travaux plus précis sur le "déjà-là » ont d'ailleurs montré qu'il agissait comme un " filtre de l'action didactique qui influencent fortement les intentions didactiques des enseignants " (Loizon, 2009). Nous nous orientons prioritairement sur son " déjà-là conceptuel " selon deux axes: la conception qu'il se fait de l'activité et celle de la transmission de son savoir. Le déjà-là conceptuel " exerce une influence constante et latente sur les démarches décisionnelles de l'enseignant d'EPS. II peut fournir à l'enseignant des motifs et mobiles possibles à la prise de décision » (Carnus, 2003, p. 11).

\subsubsection{L'épreuve}

II s'agit de la période où le sujet est " en action » et le chercheur en observation. Cette période est "un moment de vérité » (Terrisse, 1994) qui demeure néanmoins fait de mystères puisqu'il semble compliqué, comme dans toute épreuve sportive, de prévoir à l'avance ce qui va se passer, et comment la séance va se dérouler. Cette épreuve, véritable moment où le savoir se transmet, est donc constamment " soumise aux aléas de la contingence, objet d'étude privilégié en didactique » (Brousseau, 1986). Elle constitue un support décisif pour le recueil de données, puisqu'elle sera entièrement filmée, de manière à voir l'ensemble des joueurs. Nous avons équipé l'entraîneur d'un micro-cravate afin de recueillir l'ensemble de ses propos pour chaque séance afin « d'enregistrer la totalité de ce qui se passe en classe, ce qui constitue une somme d'informations considérables » (Terrisse, 2009, p. 27). L'épreuve porte sur quatre séances d'une heure trente, situées durant les mois de novembre, décembre, janvier et février. Ce choix de débuter au mois de novembre a été pensé de manière à éviter une éventuelle période d'adaptation en début d'année. À ce moment-là, les joueurs découvrent de nouvelles méthodes de travail, une rigueur et une intensité de travail plus importantes, pour s'engager dans un niveau de jeu supérieur. II s'agit donc de débuter l'épreuve au moment où les sujets ont construit des habitudes de travail et ne sont donc plus " troublés » par des facteurs extrinsèques.

Cette phase d'intervention apparaît par moment délicate pour les sujets puisqu'elle tend à dévoiler «un caractère privé et personnel »(Carnus, 2010). Dans cette perspective, l'épreuve représente un moment indispensable de l'étude puisqu'elle permet de « mettre à nu » les sujets, d'acquérir des informations singulières sur eux : sur ce qu'ils enseignent réellement et sur leurs manières de transmettre les savoirs selon les activités.

\subsubsection{L'après-coup}

L'après-coup représente le moment post épreuve et rend compte du « processus de reconstruction des évènements passés par le sujet » (Chevallard, 1985). II est utilisé dans 


\section{eJRIEPS 36 juillet 2015}

la description et la compréhension de l'entraîneur face à sa propre activité ; cela lui permet de rendre compte des savoirs qui ont été véritablement mis en jeu, et de construire, voire de reconstruire, sa séance et son enseignement au gré de l'expérience acquise. Terrisse (2007) précise que «les entretiens d'après-coup ne sont pas seulement ceux qui se déroulent après la séance. Bien entendu, le chercheur demande, en fin de séance à l'enseignant s'il a atteint ses objectifs, afin d'évaluer " à chaud " son activité. Mais nous savons, par expérience, que ce moment n'est pas toujours propice à cette évaluation, par le peu de temps que l'enseignant peut y consacrer, mais surtout parce que le moment de fin de cours laisse peu de place à l'analyse réflexive de l'enseignant ». Ce moment est donc réalisé nécessairement a posteriori, de manière à permettre au sujet de revenir sur l'événement avec un autre point de vue.

La principale méthode d'accès à "l'après-coup » est l'entretien. II reste dans une approche clinique puisqu'il permet au sujet de s'exprimer librement, d'extérioriser verbalement ce qu'il a fait, ce qu'il a pensé, ce qu'il pensait faire et ainsi de retrouver le sens de son propre enseignement. Cette verbalisation est donc un moyen fort pour le chercheur d'interpréter des sentiments, des sensations, entre les éventuels hésitations, oublis et lapsus du sujet. Ainsi, " découvrir le sens de l'acte » (Astolfi, 1993) est là tout l'intérêt de ce moment "d'après-coup " que nous avons réalisé sous forme d'entretiens post séance.

4.2. Démarche de collecte des données

Dans le cadre de cette recherche en didactique clinique, nous utilisons une méthode de recherche et de production de connaissances bien précise, déterminée par le cadre théorique de la didactique clinique. Notre sujet est l'objet d'une étude de cas, de manière à permettre l'accumulation des différentes données, afin d'en construire une conjecture, c'est-à-dire une assertion supposée mais non démontrée.

Nos résultats seront validés par la triangulation des données, méthode préconisée par différents auteurs (Van Der Maren, 1995) et définie par Lorraine Savoie-Zajq (1997). Celle-ci consiste à « adopter des postures, points de vue d'observation complémentaires, et différents types de données, puis à les confronter »(Sauvegrain, 2005, p.10). Dans cette temporalité, le recueil de données s'organise autour de plusieurs méthodes: la retranscription d'un discours d'épreuve et la retranscription d'un discours d'entretiens avant (le déjà-là) et après l'action (l'après-coup). À partir de ces méthodes, nous relevons trois types différents de données : des données de préparation, des données de discours d'épreuve, des données verbales « à chaud ». 


\section{eJRIEPS 36 juillet 2015}

\subsubsection{Les données de préparation}

Ces données représentent le discours du sujet durant des entretiens d'accès au « déjàlà », dont le but est de se centrer sur son vécu et sur ses impressions. II s'agit plus précisément de déterminer « un déjà-là en amont du processus décisionnel constitué de trois instances qui déterminent conjointement les décisions interactives : le déjà-là conceptuel, le déjà-là intentionnel et le déjà-là expérientiel (Carnus, 2003, p.17) » (Loizon, 2011). Nous avons réalisé trois entretiens d'accès au déjà-là avec Pierre dans le but de connaître, à travers des questions ouvertes, sa conception de l'activité, son « déjà-là conceptuel » à propos du basket : «quand tu enseignes le basket, qu'est-ce que tu veux enseigner à tes joueurs, de manière générale ? » « Et si on se centre un peu plus sur l'équipe U15, tu accordes une place plus particulière à quoi ? ; « Et si tu devais résumer en quelques mots la manière dont toi tu envisages le basket, de façon large, pas simplement au niveau de la formation ». Nous souhaitions aussi connaître son vécu dans l'activité à partir de questions portant sur son parcours et son expérience professionnelle. Toujours à partir de questions ouvertes, nous avons ensuite réalisé deux entretiens qui nous ont permis de préciser certains aspects de son discours, significatifs des différentes formes de son « déjà-là ». Nous avons très souvent utilisé de nombreuses relances (pas forcément sous forme de questions) au gré des réponses de notre cas : «Mais pour autant, plus le niveau va évoluer, plus la défense sera forte, plus il faudra complexifier les choses... ", (entretien $\mathrm{n}^{\circ} 1$ ), «Donc tu parles beaucoup de tout ce qui touche à la gestion... » (entretien $\mathrm{n}^{\circ} 2$ ), «Et tu n’as jamais été influencé par quoi que ce soit ? » (entretien $\left.\mathrm{n}^{\circ} 3\right)$.

\subsubsection{Les données de discours d'épreuve}

Ces données de discours d'épreuve représentent le suivi des quatre séances sur une année d'entraînement de basket-ball auprès d'une équipe évoluant en championnat de France. Nous nous sommes intéressés au discours de l'entraîneur durant l'épreuve, dans des situations à incertitudes directes, où chaque joueur est confronté à une contrainte bien précise (par exemple, « un dribble maximum », « pas de tir avant un renversement », etc). En parallèle, nous avons pris en compte le déroulement de la leçon et ses différentes phases. Ce recueil représente donc le discours de l'enseignant, que nous retranscrivons a posteriori en verbatim grâce à l'enregistrement oral par le micro-cravate.

\subsubsection{Les données verbales « à chaud »}

Ces données proviennent d'entretiens courts (d'environ cinq à dix minutes) réalisés juste après la séance, avec l'entraîneur. II s'agit ici de recueillir son point de vue, ses nouvelles 


\section{eJRIEPS 36 juillet 2015}

intentions, le bilan de son expérience sur cette séance, sous la forme d'une verbalisation orale. L'entretien, enregistré, permet de recueillir et d'analyser l'ensemble de ces informations. Cet entretien, qualifié de "non dirigé ", permet de déterminer ce que Pierre pense enseigner en terme de savoirs aux joueurs sur chaque séance, afin de le comparer à ce qu'il enseigne vraiment. Cet entretien est mené de façon à ne pas influencer ce que le sujet perçoit et ressent " à chaud ». II s'agit en effet de laisser parler librement le sujet à partir de questions très ouvertes, du type "qu'est-ce que tu penses leur avoir enseigné sur cette séance? ». Nous chercherons à constamment relancer le sujet afin de recueillir des informations sur son action qui soient les plus précises possibles.

\subsection{Le bilan de l'ensemble des données}

En conséquence, en enchaînant les entretiens avec l'entraîneur, nous avons identifié les savoirs qu'il pensait avoir enseignés : il s'agissait du savoir enseigné virtuel. L'analyse du discours de l'épreuve nous permettra de faire un bilan des savoirs qu'il a véritablement enseignés durant les séances: il s'agit là du savoir réellement enseigné. A posteriori, notre « interprétation » en tant que chercheur (Terrisse, 2009) aura pour but de déterminer s'il existe ou non un décalage entre savoir enseigné virtuel et savoir réellement enseigné, preuve de la transmission de savoirs incidents.

\section{La construction de la grille d'analyse}

\subsection{Les concepts mis en jeu}

Nous avons fait le choix de partir sur trois champs d'analyse généraux afin de construire notre grille d'analyse: les savoirs techniques, les savoirs tactiques et les savoirs méthodologiques. Chacun de ces champs est ensuite détaillé en sous-catégories qui nous permettent d'établir précisément les types de savoirs déclarés par les joueurs durant les entretiens, par l'entraîneur durant les séances, et ce afin de les comparer avec les savoirs réellement appris et enseignés.

\subsubsection{Les savoirs techniques}

Les savoirs techniques représentent l'ensemble des savoirs associés aux différents fondamentaux individuels offensifs et défensifs. II s'agit ici du " comment faire », c'est-àdire des savoirs purement moteurs, reflets des différentes actions des apprenants. P. Arnaud (1986, p.8) définit la technique comme étant « une formalisation épurée, savante et décontextualisée du savoir-faire, destiné à structurer les apprentissages ». De manière plus spécifique en basket, G. Bosc (1995, p.2) définit la technique comme « un outil dont il 


\section{eJRIEPS 36 juillet 2015}

faut savoir se servir le plus habilement possible, comme le maçon doit se servir de sa truelle ».

Ainsi, nous envisageons huit savoirs techniques bien précis dans le cadre de l'activité basket en référence à un certain nombre de productions d'experts en basket, et à ce niveau de pratique :

- les savoirs techniques sur le dribble: ils concernent «la maîtrise du rythme, la fréquence du dribble » (Vincent, 2007), «les formes du dribble » (Legrand et Rat, 1996), la hauteur, le moment du dribble, ainsi que la gestuelle à proprement parler ;

- les savoirs techniques sur la passe : ils " dépendent du moment et de la technique du lancer, mais aussi de la qualité de l'attraper » (Vincent, 2007, p.24), auxquels on peut ajouter le type, la précision et la vitesse de la passe ;

- les savoirs techniques sur le tir : ils déterminent le type, ainsi que « la tenue de la balle, l'évolution de la balle avant le lâcher et la finition du geste » (Vincent, 2007) et « l'équilibre général du corps » (Grosgeorge, 2009) ;

- les savoirs techniques sur le rebond : ils représentent «la technique de l'écran de retard » (Vincent, 2007), la présence et la hauteur du rebond ;

- les savoirs techniques sur le démarquage : ils représentent «des déplacements codifiés et structurés 》 (Vincent, 2007) et précisent le type, le moment, la gestuelle, l'amplitude, la vitesse ainsi que le « volume de jeu » du joueur (Gréhaigne, Godbout et al, 1997);

- les savoirs techniques sur la feinte : ils définissent « la mobilisation du pied tireur, l'amplitude et la vitesse » (Vincent, 2007), le type, le rôle, la vitesse, l'amplitude, le rythme et le moment de la feinte ;

- les savoirs techniques sur la défense : ils précisent les déplacements et la prise d'information (Vincent, 2007), la gestuelle, le type (sur porteur de balle, sur non porteur de balle), la forme (homme à homme, zone, zone press), le but, le moment et le rythme de la défense ;

- les savoirs techniques sur les appuis : ils représentent aussi bien «technique de course, équilibre, arrêt, pivot, départ, changement de direction » (Noteboom, 2004) ainsi le type, la vitesse, la fréquence et l'orientation des appuis ;

- Les savoirs techniques sur les écrans : ils caractérisent «les principes liés à son statut de poseur ou de receveur d'écran » (Lehmann, 1996), le type, l'orientation et la façon d'utiliser les écrans. 


\section{eJRIEPS 36 juillet 2015}

\subsubsection{Les savoirs tactiques}

Les savoirs tactiques et stratégiques, mettent en jeu le "quoi faire" de l'apprenant. Georges (1983, p. 22) définit ce type de savoirs comme étant un « ensemble de principes directeurs susceptibles de diriger l'élaboration d'une procédure, en raison de la présence stable de règles de décision et d'action permettant de rendre compte des choix effectués ». Il s'agit donc bien de l'ensemble des moyens, des démarches, des intentions et des stratégies développés pour atteindre un objectif fixé par l'entraîneur. Les mécanismes de prises de décision, de choix et de mises en œuvre cognitives seront ici centraux. D'un point de vue plus spécifique au basket, la tactique, "c'est savoir quoi faire de ses habiletés et comment éventuellement les adapter ou les masquer pour tromper l'adversaire » (Bosc, 1995, p. 2).

Dans cette perspective, elle repose sur trois notions majeures :

- la lecture du jeu: Ciani (1997) estime que "la chose la plus importante est d'augmenter la capacité des joueurs à lire une situation », afin de " trouver les réponses techniques appropriées au problème posé par la défense ou d'une façon plus générale savoir, par ses actions, poser problème à l'adversaire 》 (Grosgeorge et Wolff, 1998, p.11) ;

- la prise de décision : pour I. Ballarini et B. Grosgeorge (1996, p. 3), "L'élaboration de la base décisionnelle constitue une véritable base d'orientation de l'action, elle se construit progressivement, elle doit être mise d'emblée en relation étroite avec la dimension technique du geste »;

- la gestion du collectif : pour J-F. Gréhaigne, M. Billard et J-Y. Laroche (1999, p. 51), « le propre du joueur de sport collectif réside dans la capacité à détecter, en plein jeu, les évolutions naissantes dans toute la complexité du rapport d'opposition, à en déduire les choix d'action successivement appropriés au devenir du jeu et qui se matérialisent d'instant en instant sur le terrain ». Ainsi, visualiser le rapport de force, l'analyser et le prendre en compte afin de mettre en œuvre des réponses motrices efficaces constituent la gestion tactique et son importance.

\subsubsection{Les savoirs méthodologiques}

Les savoirs méthodologiques représentent l'ensemble des éléments à prendre en compte pour être efficace et importants au sein du collectif et reposent sur trois notions prioritaires : 


\section{eJRIEPS 36 juillet 2015}

- la connaissance du règlement, puisque les règles sont « les conditions du jeu garantissant l'égalité des chances de chaque protagoniste » (Méard et Bertone, 1996, p. 16) ;

- la communication, qui doit être efficace afin d' "exploiter une défaillance individuelle, la moindre faute d'inattention des adversaires ou encore les forcer à commettre des erreurs » (Gréhaigne, Billard, Laroche, 1999, p. 51) ;

- le leadership, en lien notamment avec l'importance du meneur du jeu dans le collectif et puisque "la cohésion de l'équipe est réalisée par l'adhésion et le partage de valeurs explicites sur lesquels se fondent les décisions des leaders » (Mazer, 2002, p. 8).

\subsection{La Grille d'analyse}

Nous proposons au lecteur une grille d'analyse de l'ensemble des données, que nous présentons en annexe 1 sous forme de tableau-bilan, indispensable pour mieux comprendre la suite de l'article.

\section{Les résultats de l'étude}

6.1. L'analyse des différentes données

Notre analyse est réalisée en plusieurs temps. Un premier temps d'analyse des entretiens de préparation réalisés avec Pierre, afin de mettre à jour un « déjà-là intentionnel général » pour tenter d'approcher le « déjà-là conceptuel » et le "déjà-là expérientiel ». Le deuxième temps d'analyse de son discours durant les séances, doit permettre de déterminer quantitativement les savoirs enseignés durant les séances. Enfin, un troisième temps d'analyse des entretiens post-séance, pour préciser l'ensemble des savoirs qu'il pense avoir enseigné.

\subsubsection{L'analyse des entretiens d'accès au déjà-là}

De l'ensemble de ces entretiens, on peut relever plusieurs points importants concernant la manière dont Pierre envisage l'activité et son enseignement. II s'agit donc de la détermination progressive de son déjà-là.

Sa priorité dans son enseignement, son « déjà-là intentionnel », face à des jeunes joueurs repose sur deux aspects : la maîtrise technique et le collectif. Enseigner l'activité, c'est d'abord enseigner les bases techniques, les fondamentaux, puisque c'est à partir d'eux que les joueurs pourront se mouvoir dans un projet collectif bien défini : « La technique de tir, de dribble, de passe, les vrais fondamentaux quoi. C'est cette maitrise technique qui doit être essentielle. Offensivement je parle. Savoir être techniquement capable d'attraper- 


\section{eJRIEPS 36 juillet 2015}

tirer, attraper-passer, désaxer les appuis, désaxer les bras, c'est une maîtrise technique qui est la base " (entretien $n^{\circ} 3$ ). En outre, le collectif pour lui revêt une importance capitale puisqu'il va permettre de contrecarrer n'importe quelle décision adverse et permettre à chacun, "de se montrer, d'avoir une réelle influence " (entretien $\mathrm{n}^{\circ} 1$ ), afin "d'être capable d'enchaîner encore plus les mouvements, être capable d'avoir une meilleure harmonisation des mouvements, un meilleur timing de travail ensemble" (entretien $n^{\circ} 3$ ).

Trois notions récurrentes sont au centre de son enseignement de l'activité et déterminent son « déjà-là conceptuel »: la simplicité, l'intensité et l'efficacité. On les relève dans le premier entretien : "Notre basket doit être un basket intense, et ça à tout niveau. Et si on est intense, on doit rechercher une efficacité. Et la simplicité oui, car il ne faut pas se compliquer la vie. Si on est intense, il ne faut pas s'emballer à faire des choses un peu aléatoires. Il vaut mieux être simple : si tu es simple, tu es efficace 》 (entretien n¹).

Dans la formation, il va chercher à construire des joueurs malins et "vicieux » (entretien $n^{\circ}{ }^{1}$ ), qui représentent deux notions très fortes pour lui. Malin puisque «le joueur doit être capable de gérer son groupe, son équipe, gérer le jeu, il est intelligent dans le jeu, sur les moments de jeu, en fonction du score, en fonction de l'adversaire, combien il a de fautes, il est capable de sanctionner directement. Le joueur malin c'est ça, il doit bien contrôler tout le jeu » (entretien $n^{\circ} 2$ ). Vicieux afin de jouer sur tout «ce que ne voit pas l'arbitre: tirer un peu le short, mettre la main sur la tronche pendant le tir, sortir les coudes quand il faut, un petit peu tout ça, mais dans la connaissance de où est l'arbitre " (entretien n²). La singularité du sujet s'exprime donc dans ses différents « déjà-là ».

\subsubsection{L'analyse du discours de séance}

Afin d'analyser les propos de Pierre, nous les avons triés par type de savoir, c'est-à-dire qu'à chaque fois qu'une acception se rapprochait d'un savoir précis, nous le catégorisions dans un type de savoir particulier. Par exemple, lorsque Pierre déclare "Quand vous sortez sur le step, mettez vous parallèle à la ligne de touche " (discours de la séance $n^{\circ} 2$ ), il enseigne un savoir technique sur la défense (STDéf) et donc concrètement, dans notre analyse du discours de la séance $n^{\circ} 2$, nous notons un nouveau savoir technique sur la défense. L'intérêt de cette retranscription est ainsi de déterminer concrètement ce que Pierre a enseigné en terme de savoirs et d'objets de savoirs durant la séance. 


\section{eJRIEPS 36 juillet 2015}

Dès lors, nous présentons en annexe 2 un bilan quantitatif de l'ensemble des objets de savoirs dispensés par Pierre durant chaque séance. Nous illustrons ces tableaux à partir de diagrammes apportant plus de visibilité à nos résultats statistiques.

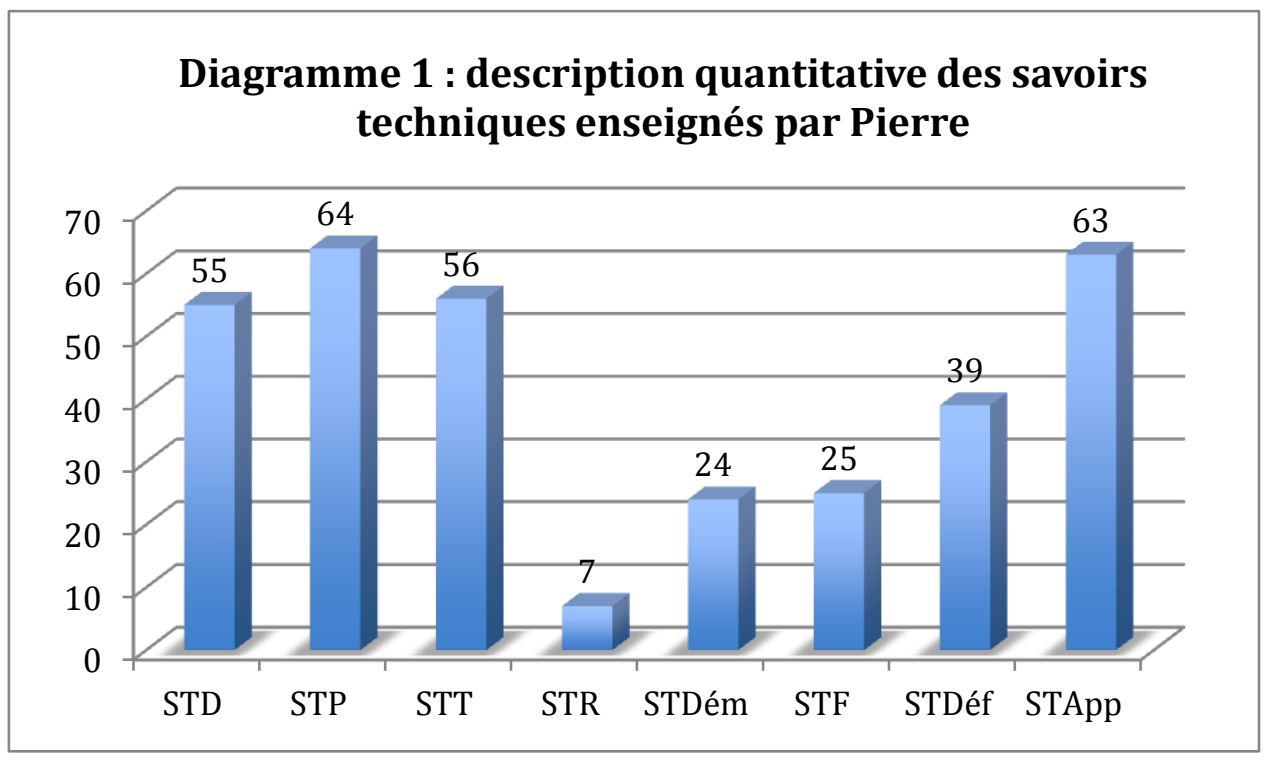

Ce diagramme 1 illustre les savoirs techniques enseignés par Pierre : on remarque que les savoirs techniques sur le dribble (STD), sur la passe (STP), sur le tir (STT) et sur les appuis (STApp) apparaissent prioritaires dans son discours de séance (confère " annexe 1 » pour comprendre l'ensemble des sigles).

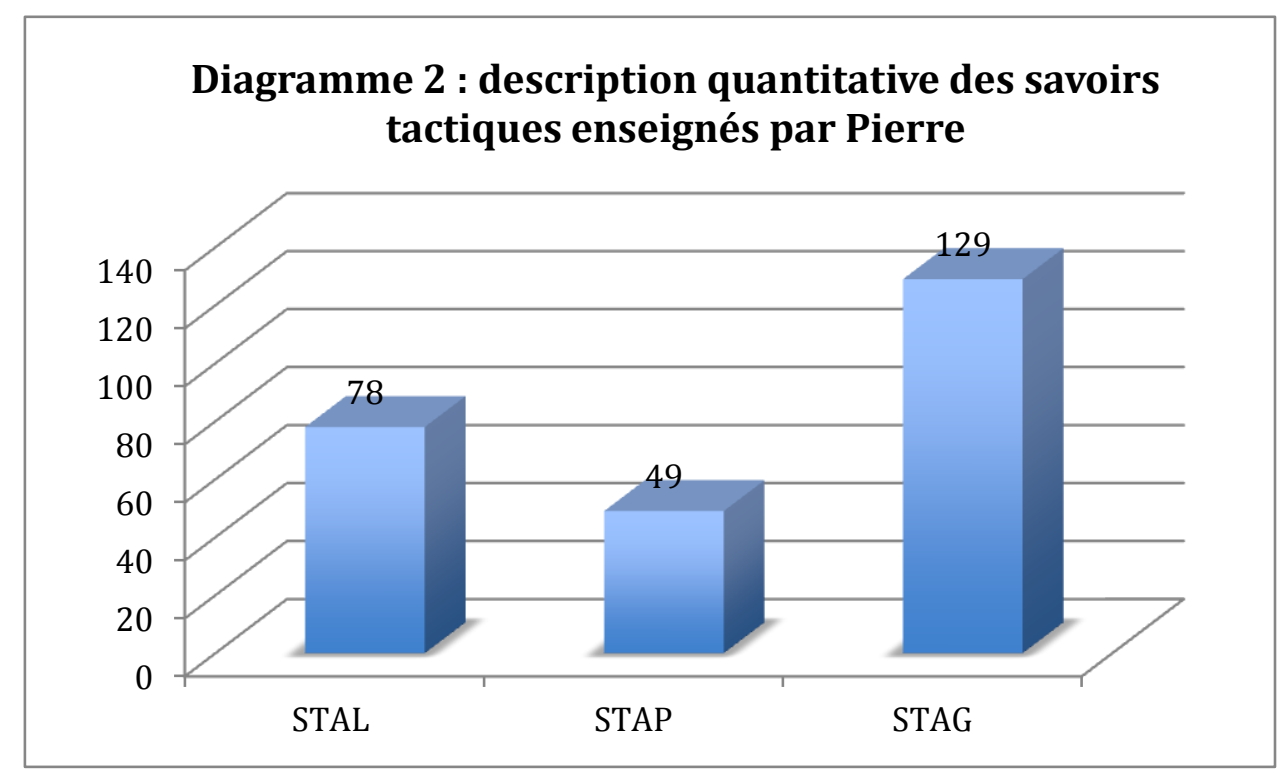

Le diagramme 2 illustre les savoirs tactiques enseignés par Pierre durant sa séance. On constate que les savoirs tactiques sur la gestion tactique (STAG) et sur la lecture du jeu 


\section{eJRIEPS 36 juillet 2015}

(STAL) sont centraux et laissent les savoirs tactiques sur la prise de décision (STAP) à la marge (confère annexe 1).

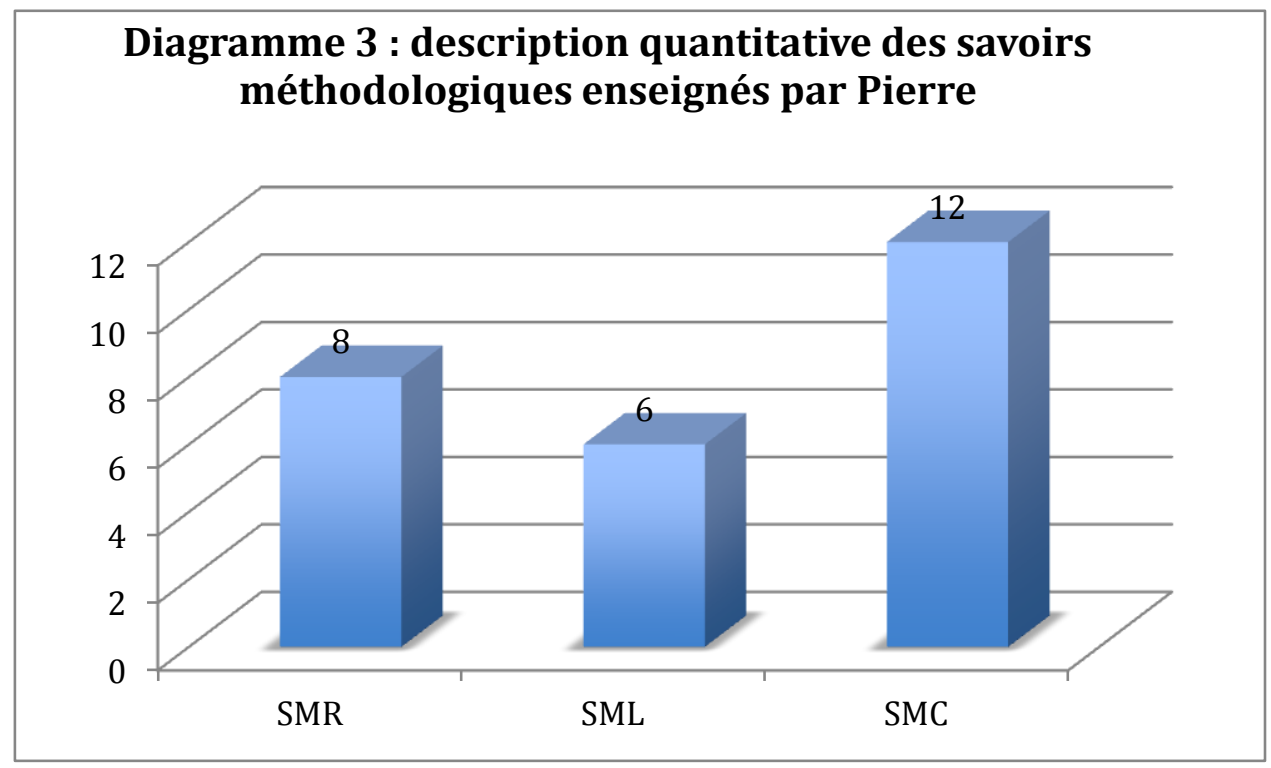

Le diagramme 3 atteste des savoirs méthodologiques enseignés par Pierre et montre que les savoirs méthodologiques sur la communication (SMC) sont prioritairement enseignés ; ceux sur le règlement et le leadership étant moins présents.

Ainsi, à partir de ces constats, nous pouvons proposer certaines analyses.

N.B. : précisons que l'ensemble des objets de savoirs présents dans les discours de séance ne représente pas des objets de savoirs uniques enseignés par Pierre, mais la totalité des objets de savoirs qui ont pu être enseignés, donc parfois, cela peut représenter le même objet de savoir, répété plusieurs fois sur plusieurs séances.

Le nombre d'objets de savoirs transmis par Pierre aux joueurs à chaque séance est conséquent : il transmet entre 105 et 253 objets de savoirs à chaque séance. C'est un entraîneur qui régule constamment l'activité des joueurs et qui individualise un maximum ses feedbacks.

De plus, nous constatons que Pierre transmet aux joueurs énormément d'objets de savoirs, mais très souvent, il s'agit de savoirs identiques, qui sont répétés au gré de la performance motrice des jeunes. Par exemple, lors de la première séance, il transmet quinze fois le même savoir à propos de la cible : «Regarde le cercle ». Ces régulations interviennent souvent comme rappel de ce qu'il faut faire techniquement et tactiquement 


\section{eJRIEPS 36 juillet 2015}

et sur lesquels il fait le choix d'insister énormément : « soit malin », « ne regarde pas ta balle », «fléchis» sont trois objets de savoirs que Pierre répète constamment sur l'ensemble des quatre séances. De plus, ces savoirs peuvent être très souvent extrêmement précis et représentent un détail de ce qui est attendu chez les joueurs. On note notamment sur la deuxième séance des objets de savoir très précis tels que : «C'est je passe dessus, et je vais faire en sorte de défendre comme un mort de faim. Je passe au-dessus et proche. Faut pas reculer là » et « Repousse comme ça, parallèle à la ligne ».

L'analyse du discours de ces séances montre que Pierre transmet plusieurs types de savoirs et ne se restreint pas aux savoirs propres aux thèmes des séances. En effet, sur 14 types de savoir au total définis dans notre grille d'analyse, il en dispense en moyenne 11,3 par séance (10 à la première, 12 à la deuxième, 11 à la troisième et 12 à la quatrième). En outre, en faisant un focus sur les savoirs techniques, on constate qu'il transmet en moyenne 6,5 types de savoirs (5 pour la séance 1 et 7 pour les séances 2, 3 et 4). Néanmoins, son enseignement se centre sur des objets de savoirs précis et liés à ses thèmes de séances : pour la séance 1 dont le thème était « la fixation des intervalles et l'ouverture des angles de passes » (entretien post-séance $\mathrm{n}^{\circ} 1$ ), il dispense 59 objets de savoirs sur la gestion tactique («Ils arrivent tous les deux, je ne vais pas aller au milieu, je vais peut être m'écarter ») et 26 sur la passe ( «tendez les bras ») ; pour la séance 2 dont le thème était «les passes, les finitions et la défense sur écran » (entretien post-séance $n^{\circ} 2$ ), il enseigne 25 objets de savoirs techniques sur la passe (la régulation « tendez les bras » est toujours très présente), 15 sur le tir (« le coude au niveau de l'épaule ») et 26 sur la défense (« reprenez vos joueurs ») ; pour la séance 3 dont le thème était «la prise d'information » (entretien post-séance $n^{\circ} 3$ ), il enseigne 31 objets de savoirs sur la lecture du jeu (« Dans une aisance technique, le dribble est de dextérité et surtout dans un regard porté sur tout sauf le ballon») et 37 sur la gestion tactique ( Passe arrière arrière, on switch, après passe ailier arrière, l'écran par le bas ») ; enfin pour la séance 4 dont le thème était « la qualité de la passe » (entretien post-séance $n^{\circ} 4$ ), il dispense 13 objets de savoirs techniques sur la passe («Vraiment plus hautes vos passes les garçons») et 22 sur la gestion tactique ( Renverse cette balle»). L'ensemble de ces résultats montre bien la relation entre le thème général de la séance et les types d'objets de savoirs enseignés prioritairement ainsi que les autres savoirs moins prioritaires mais présents. 


\section{eJRIEPS 36 juillet 2015}

On constate également une forte prédominance des savoirs techniques transmis, en comparaison avec les savoirs tactiques et méthodologiques. En effet, le bilan global montre que Pierre a enseigné en totalité sur les quatre séances 333 objets de savoirs techniques, 256 tactiques et seulement 26 méthodologiques. Pierre intervient en priorité sur la maîtrise technique de chaque joueur, en apportant de nombreuses régulations, toujours très précises, comme nous l'avons montré dans le deuxième point de ce bilan. Néanmoins, il n'oublie jamais de relier les objets de savoirs techniques avec les objets de savoirs tactiques, en associant régulièrement deux savoirs technique et tactique, comme par exemple " il faut que les passes aillent vite pour créer un décalage dans la défense " (discours $n^{\circ} 3$ ) : là, Pierre met en évidence la vitesse des passes avec la conséquence sur la défense. On constate également à chaque séance un nombre d'objets de savoirs tactiques enseignés globalement inférieur aux objets de savoirs techniques :

\begin{tabular}{|l|l|l|}
\hline & $\begin{array}{l}\text { Nombre d'objets de } \\
\text { savoirs techniques }\end{array}$ & $\begin{array}{l}\text { Nombre d'objets de savoirs } \\
\text { tactiques }\end{array}$ \\
\hline Séance 1 & 130 & 114 \\
\hline Séance 2 & 79 & 18 \\
\hline Séance 3 & 61 & 80 \\
\hline Séance 4 & 63 & 44 \\
\hline
\end{tabular}

Les savoirs techniques enseignés ne sont pas centrés sur un type donné mais sont assez globaux puisqu'on retrouve des proportions proches au niveau des savoirs sur le tir (56), la passe (64), le dribble (55) et les appuis (63). Concernant les savoirs tactiques, cette remarque n'est plus valable puisque Pierre enseigne beaucoup plus d'objets de savoirs sur la gestion tactique (129) que sur la lecture du jeu (78) et la prise de décision (49). Ceci est en relation directe avec sa conception de l'activité et de la formation puisqu'il conçoit le joueur de basket-ball comme un joueur qui doit d'abord "être capable de gérer son groupe, son équipe, gérer le jeu » (entretien $n^{\circ} 1$ ).

Le nombre d'objets de savoirs méthodologiques enseignés aux joueurs est très faible, voire presque négligeable comparés aux savoirs techniques et tactiques. Pierre insiste très peu sur le rôle de leadership ou sur les aspects du règlement. La communication apparaît néanmoins quelquefois dans les savoirs transmis aux joueurs, notamment concernant la défense, par des régulations du type «l'intérieur il t'annonce droite et en fait 


\section{eJRIEPS 36 juillet 2015}

tu vas glisser, c'est à dire que tu vas passer entre le joueur qui porte l'écran et moi » et « si on fait dessous, tu annonces glisse ou dessous 》 (discours n²).

\subsubsection{L'analyse des entretiens post-séance}

Pour analyser le discours de ces entretiens, nous avons choisi la même grille d'analyse et la même méthode de recueil des données afin de pouvoir facilement comparer les savoirs enseignés virtuels avec les savoirs enseignés réellement. Nous présentons les résultats obtenus séance par séance en annexe 3.

Au regard de ces résultats, le bilan que nous pouvons faire sur les savoirs enseignés virtuels se résume en plusieurs points. Hormis à la deuxième séance, Pierre pense enseigner à peu près toujours autant de savoirs (entre 15 et 20, avec une moyenne de 19 savoirs par séance).

Les résultats apparaissent très hétérogènes : sur certaines séances, le nombre de savoirs techniques est supérieur au nombre de savoirs tactiques, alors que sur d'autres, c'est le contraire. Par exemple sur les séances 2 et 4 , il pense enseigner plus de savoirs techniques que tactiques (17 et 12 pour 4 et 7), avec notamment «passer proche de l'épaule et être capable d'emmener le joueur avant» (entretien d'après-coup n²) et « prendre la balle au-dessus de la main et la fouetter avec le poignet rapidement et qu'ils comprennent que c'est vraiment une accélération avant bras poignet » (entretien d'aprèscoup $\left.\mathrm{n}^{\circ} 4\right)$. Au contraire, sur les séances 1 et 3 , il pense enseigner plus de savoirs tactiques que techniques (7 et 14 pour 6 et 3), dont par exemple « c'est dans la qualité de la fixation et de trouver le bon choix de passe » (entretien d'après-coup $n^{\circ} 1$ ) et « donc soit monter la balle rapidement pour éviter la pression, soit avoir une collaboration à deux comme on fonctionne à deux arrières » (entretien d'après-coup $n^{\circ} 4$ ).

Sur l'ensemble des séances, il pense enseigner plus de savoirs techniques (48), du type « faire des passes rapidement, sèches et vite » (entretien d'après-coup n4) et « ensuite c'est dribbler en étant très fléchie et limiter le nombre de dribbles » (entretien d'après-coup $\left.n^{\circ} 4\right)$ que de savoirs tactiques (32), comme « ne plus être centré sur sa balle mais plutôt sur tout ce qui est autour » (entretien d'après-coup n`3) et « d'alterner le jeu de fixation et de passes, fixation par le drive et le jeu de passes " (entretien d'après-coup n`3) et méthodologiques (7), avec par exemple «être capable de communiquer» (entretien d'après-coup $\mathrm{n}^{\circ} 2$ ) et « les arbitres vont siffler écran mobile, car il faut le temps que le défenseur ait le temps de tourner la tête pour voir l'écran » (entretien d'après-coup n²). 


\section{eJRIEPS 36 juillet 2015}

Pierre a l'impression de vraiment cibler les savoirs qu'il transmet aux joueurs lors de ses entraînements. II n'évoque que quelques types de savoirs bien précis, comme "cadrer les appuis du joueur entre $50 \mathrm{~cm}$ et $1 \mathrm{~m}$ ” (entretien d'après-coup $\mathrm{n}^{\circ} 2$ ) et non un large éventail : 4 types de savoirs pour la séance 1, 8 pour la séance 2, 5 pour la séance 3 et 5 pour la séance 4 , sur les 14 types de savoirs au total, soit une moyenne de 5,5 types de savoirs par séance.

Ainsi, ces entretiens post séance nous ont permis de déterminer "les raisons de l'acte didactique » (Carnus, 2009), qui dans notre étude se traduit par les savoirs que Pierre enseigne "virtuellement". A partir de là, nous sommes en mesure de comparer précisément ces savoirs avec ceux qu'il a enseignés réellement.

6.2. La comparaison des deux types de données

Après avoir présenté et analysé l'ensemble des données, nous allons comparer les données qui semblent, en certains points, assez différentes.

\begin{tabular}{|c|l|l|}
\hline & \multicolumn{1}{|c|}{$\begin{array}{c}\text { Savoirs réellement } \\
\text { enseignés }\end{array}$} & Savoirs enseignés virtuels \\
\hline $\begin{array}{c}\text { Quantification des types } \\
\text { de savoirs }\end{array}$ & $\begin{array}{l}\text { En moyenne : 11,3 types } \\
\text { par séance }\end{array}$ & En moyenne : 5,5 types \\
\hline Les savoirs techniques & $\begin{array}{l}\text { En moyenne : 6,5 types } \\
\text { par séance }\end{array}$ & $\begin{array}{l}\text { En moyenne : 2,75 par } \\
\text { séance }\end{array}$ \\
\hline Les savoirs tactiques & $\begin{array}{l}\text { Centrés sur la gestion } \\
\text { tactiques (129) contre 78 } \\
\text { pour la lecture du jeu et 49 } \\
\text { pour la prise de décision }\end{array}$ & $\begin{array}{l}\text { Centrés sur la lecture du } \\
\text { jeu (16 contre 5 pour la } \\
\text { prise de décision et 11 } \\
\text { pour la gestion tactique) }\end{array}$ \\
\hline
\end{tabular}

Ainsi, dans un objectif d'analyse et au regard de ces éléments de comparaison entre les savoirs réellement enseignés et les savoirs enseignés virtuels, nous constatons que Pierre enseigne réellement plus de types de savoirs qu'il ne le pense, comme l'a montré Loizon (2004) pour les professeurs de judo sur les variables didactiques. Son enseignement est beaucoup plus vaste qu'il ne l'imagine puisqu'il enseigne des savoirs qui ont trait à un plus grand nombre de catégories. Ensuite, il enseigne des savoirs techniques de tous types alors qu'il pense se centrer sur des savoirs techniques précis. II cible donc beaucoup 


\section{eJRIEPS 36 juillet 2015}

moins les savoirs techniques réellement enseignés puisque ses interventions ne sont pas centrées sur le but technique de la situation mais se dispersent sur tout ce qu'il peut constater en terme de conduites. Enfin, cet entraineur propose en priorité des savoirs tactiques sur la gestion du jeu alors qu'il pense enseigner en priorité des savoirs tactiques sur la lecture du jeu. Les savoirs réellement enseignés ne correspondent pas toujours avec les savoirs enseignés virtuels. En d'autres termes, on constate qu'il existe bel et bien un écart entre les savoirs réellement enseignés et les savoirs enseignés virtuels, puisqu'il existe des savoirs enseignés dont il ne parle pas dans les entretiens, que l'on pourrait plus largement qualifier de savoirs incidents. De plus, cet écart montre que cet entraîneur de basket-ball professionnel enseigne des savoirs techniques qu'il n'envisageait pas forcément, et des savoirs tactiques qui n'étaient pas dans ses priorités énoncées.

\subsection{La trace d'une division}

La triangulation de certaines données permet de mettre en évidence chez Pierre la trace d'une division entre deux formes de basket: d'un côté le basket américain, plutôt morphocinétique, avec une volonté de spectacularisation, et de l'autre côté, un basket plus européen centré sur l'efficacité individuelle et collective, et beaucoup moins spectaculaire (Archambault, Artiaga et Bosc, 2005).

\begin{tabular}{|l|l|l|}
\hline Données & Extraits & Commentaires \\
\hline De préparation & $\begin{array}{l}\text { "Je préfère un meneur cérébral } \\
\text { qu'un meneur physique » } \\
\text { «Si je pars sur un fondement } \\
\text { collectif, je veux des gens un peu } \\
\text { intelligents » }\end{array}$ & $\begin{array}{l}\text { Extrait du premier } \\
\text { entretien de préparation }\end{array}$ \\
\hline $\begin{array}{l}\text { De discours de de } \\
\text { séance }\end{array}$ & $\begin{array}{l}\text { «Alors pourquoi tu dribbles sur } \\
\text { place, je te demande de poser des } \\
\text { dribbles vers le cercle moi. » }\end{array}$ & $\begin{array}{l}\text { Extrait de verbatim au } \\
\text { cours de la séance 1. }\end{array}$ \\
\hline Post-séance & $\begin{array}{l}\text { "Pas forcément être le plus fort, } \\
\text { avec 12 dribbles entre les jambes } \\
\text { ou dans le dos, mais c'est vraiment } \\
\text { ne pas perdre le ballon » } \\
\text { «Si le ballon vit, à un moment ça } \\
\text { s'aère » }\end{array}$ & $\begin{array}{l}\text { Extrait de l'entretien } \\
\text { post-séance n³. }\end{array}$ \\
\hline
\end{tabular}

Cette division de l'entraîneur tiraillé entre ces deux formes de basket traduit une envie de se rapprocher d'un basket plus européen. En effet, les extraits relevés ci-dessus montrent qu'il cherche à construire un joueur collectif, qui vit plus par la passe que par le dribble et qui comprend le jeu. L'option de développer ce type de basket chez les jeunes s'avère plus contraignant puisqu'il tend à développer des capacités cognitives plus importantes. 


\section{eJRIEPS 36 juillet 2015}

\subsection{La singularité du sujet au travers de ses déjà-là}

Son déjà-là expérientiel est exprimé dans son histoire professionnelle de coach : " Je pense que je me suis formé en tant que joueur en me formant en tant que coach [...] Et en fait, je me suis vraiment formé en tant que joueur et démonstrateur quand j'entraîne avec les autres qui m'entourent, les entraîneurs avec qui j'ai pu travailler, au pôle, sur les sélections. [...] Après tu fais le tri, dans ton basket, de ce que toi tu prends, de ce qui te correspond, de ce que tu veux, c'est à dire pour moi un basket à la base collective où chaque joueur a un rôle et un basket où tu maîtrises techniquement les choses et qui va te permettre de développer ce collectif » (discours d'accès au déjà-là $n^{\circ} 1$ ). Ces propos de Pierre résument son histoire d'abord en tant que joueur, puis en tant qu'entraîneur en montrant la relation très forte qui existe entre expérience de joueur et construction de sa conception de la formation du joueur : il s'agit bien là de ce qu'on nomme en didactique clinique les déjà expérientiel et conceptuel.

Nous avons montré que durant son enseignement, Pierre convertit ces deux formes de déjà-là à travers des savoirs techniques enseignés prioritairement (reflet de la maîtrise technique) mais aussi des savoirs tactiques centrés sur la gestion du jeu (reflet de l'aspect collectif de l'activité) : on peut donc véritablement parler dans notre étude d'une réelle " conversion didactique » (Buznic-Bourgeacq, 2005). Dans son activité d'entraineur, il cherche à transmettre ce qu'il a vécu et construit en tant que joueur.

\section{Conclusion}

Les savoirs enseignés par cet entraineur de basket sont donc très nombreux si l'on se réfère au grand nombre d'objets de savoirs que nous avons définis dans notre recherche ; cette multitude de savoirs est associée à une grande variété dans chacune des séances observées. Néanmoins, l'entraîneur se centre sur le thème de sa séance de manière à développer prioritairement des savoirs bien spécifiques à chaque séance, même si d'autres objets de savoir apparaissent aussi.

Bien que le savoir spécifique au basket soit dissocié (entre savoir technique, tactique et méthodologique) pour les besoins de notre étude, il n'en demeure pas moins que les savoirs tactiques et techniques sont constamment reliés, même si la priorité dans l'enseignement repose sur des savoirs techniques, indispensables pour construire chez l'apprenant les outils pour se mouvoir au sein d'un collectif. Malgré tout, l'apport de savoirs méthodologiques semble négligeable puisqu'ils sont quasiment ignorés durant l'enseignement, l'entraîneur privilégiant la transmission de savoirs techniques et tactiques, 


\section{eJRIEPS 36 juillet 2015}

se centrant plus sur le " comment faire » et le "pourquoi faire ", que sur l' "être » et « savoir-être ».

Enfin, il existe un réel écart entre les savoirs réellement enseignés et virtuels tendant à montrer la trace d'enseignements incidents ; c'est bien cela qui rend si compliqué cet acte qu'est l'enseignement sportif.

\section{Bibliographie}

Archambault, F., Artiaga, L., \& Bosc, G. (2005, Novembre). Double jeu: histoire du basket-ball entre France et Amériques. Paris.

Astolfi, J. P. (1993). Trois paradigmes pour les recherches en didactique. Revue Française de Pédagogie, 113. (pp. 5-18). Paris : INRP.

Astolfi, J. P. (2008). La saveur des savoirs. Discipline et plaisir d'apprendre. Paris : ESF.

Ballarini, I., \& Grosgeorge, B. (1996). Lire le jeu - Jouer juste. Basket-ball, 614.

Beillerot, J. (1989). Voies et voix de la formation. Paris : Editions Universitaires.

Bosc, G. (1995). L'Essentiel. Basketball, 605, 1-3.

Bouthier, D., \& Durey, A. (1994). Technologie des APS. Impulsions, 1, 95-124. Paris : INRP.

Buznic-Bourgeacq, P. (2005). L'expérience de l'enseignant et ses implications didactiques : une étude de cas en EPS. 5e colloque international Recherche et Formation, Former des enseignants professionnels, savoirs et compétences. IUFM des pays de la Loire.

Buznic-Bourgeacq, P., Terrisse, A., \& Margnes, E. (2010). La transmission du savoir expérientiel en EPS : études de cas et analyses comparatives en didactique clinique [en ligne]. eJRIEPS, 20, 26-47.

Carnus, M. F. (2003). Analyse en didactique du processus décisionnel de l'enseignant d'EPS en gymnastique : une étude de as croisés, in C. Amade-Escot, Didactique de l'éducation physique, état des recherches, (pp. 193-224). Paris : Revue EPS.

Carnus, M.-F. (2010). L'inscription clinique en didactique de l'éducation physique et sportive, Cliopsy, n 4, 73-88.

Chevallard, Y. (1985). La transposition didactique: du savoir savant au savoir enseigné. Grenoble : La Pensée Sauvage.

Chevallard, Y. (1991). La transposition didactique, du savoir savant au savoir enseigné. Grenoble : La Pensée Sauvage. 


\section{eJRIEPS 36 juillet 2015}

Delignières, D., Duret P. (2004). Lexique thématique en sciences et techniques des activités physiques et sportives. Paris : Vigot.

Garnier, A. (2008). Le rapport au savoir de l'enseignant, révélateur du fonctionnement du système didactique [en ligne]. eJRIEPS, 14, 22-40.

Georges, C. (1983). Apprendre par l'action. Paris : PUF.

Gréhaigne, J.F., Godbout, P., \& Bouthier, D. (1997). Performance assessment in team sports. Journal of Teaching in Physical Education, 16, 500-516.

Gréhaigne, J-F. (1999). Quels référents théoriques pour la modélisation en football.

Grosgeorge, B., \& Wolff, M. (1998). Petit lexique du joueur de basket. Basket-Ball, 602.

Grosgeorge, B., (2009). Le tir de champ au basket, approche biomécanique et neurophysiologique. Pivot, 139, 26-28.

Heuser, F., Terrisse, A., \& Carnus, M.F. (2005). Ecart entre savoir enseigné et savoir appris en éducation physique et sportive : étude de cas en karaté [en ligne]. eJRIEPS, 7, 57-74.

Joshua, S. (1996) Le concept de transposition didactique n'est-il propre qu'au mathématiques ?, in Raisky, C. et Caillot, M. (dir) Au-delà des didactiques, le didactique. Débats autour de concepts fédérateurs, Bruxelles, De Boeck, pp. 6173.

Legrand, L., \& Rat, M. (1996) Le basket-ball, les règles - la technique - la pratique. Toulouse : Milan.

Lehmann, E. (1996). Pose et utilisation des écrans. Basket-ball, 611, 1-3.

Loizon D., Margnes, E et Terrisse A. (2008) Analyse des pratiques d'enseignement du judo en EPS. Ejrieps n¹4, IUFM de Besançon, 63-83.

Loizon, D. (2009). Les filtres personnels dans l'action didactique, ouvrage coordonné par A. Terrisse sur la Didactique clinique. Bruxelles : De Boeck.

Loizon D., Carnus M.-F. (2011) Le déjà-là décisionnel chez les enseignants novices en EPS. Un outil pour comprendre la réalité de leurs enseignements, Symposium REF « Construction de l'identité professionnelle chez des stagiaires et des enseignants novices en éducation physique »Louvain la Neuve, 13 et 14 septembre.

Malglaive, G. (1987). Enseigner à des adultes. Paris : PUF.

Mazer, J-E. (2002). Teamthink - management d'équipe clé du succes. Paris : INSEP-Publ.

Méard, J., \& Bertone, S. (1996). L'élève qui ne veut pas apprendre en EPS : propositions pour rendre l'activité de l'élève signifiante. EP\&S, 259, 61-64. 


\section{eJRIEPS 36 juillet 2015}

Noteboom, T. (2004). Sport transversal et éducation à la "socio-motricité » [en ligne]. Clés pour la forme, dossier Handball, 6, 1-5.

Raynal, F. et Rieunier, A. (1997). Pédagogie, dictionnaire des concepts clés. Paris : ESF.

Sauvegrain, J. P. (2001). Analyse didactique de la décision de l'élève, étude de cas sur l'utilisation du savoir dans un cycle de lutte en Education Physique et Sportive. Thèse non publiée, Université Paul Sabatier Toulouse III.

Sauvegrain, J. P. (2005). La prise en compte du sujet élève dans la recherche sur l'appropriation du savoir par les élèves de 5è au cours d'un cycle de lutte. In le symposium animé par A Terrisse, la question du sujet en didactique de l'EPS, Colloque de l'Aris, Intervenir dans les PAS, pratiques, recherches, formations. Louvain-la-Neuve : Belgique.

Savoie-Zacq, L. (1996). Triangulation. In A. Muchielli. Dictionnaire des méthodes qualitatives en sciences humaines et sociales. (pp. 261-262). Paris: Armand Colin.

Terrisse, A. (1994). La question du savoir dans la didactique des APS. Habilitation à diriger des recherches. Université Paul Sabatier. Toulouse III.Terrisse, A. (1999). La question du rapport au savoir dans le processus d'enseignementapprentissage : le point de vue de la clinique. (pp.62-87). Amiens : Carrefours de l'éducation 7.

Terrisse A. (2000) Épistémologie de la recherche clinique en sports de combat, in A. Terrisse (sous la direction de), Recherches en Sports de combat et Arts Martiaux, État des lieux (pp. 95-108). Paris : Éditions revue EP.S.

Terrisse, A. (2007). Rapport au savoir et enjeux de savoir pour des enseignants d'EPS : « Une médiation aisée pour situer le sujet : d'un rapport au savoir ». Colloque de l'AECSE. Strasbourg, septembre 2007.

Terrisse, A. et Carnus, M. F. (2009). Didactique clinique de l'éducation physique et sportive (EPS), quels enjeux de savoirs. Paris : De Boeck.

Van der Maren, J. M. (1995). Méthodes de recherche pour l'éducation. Bruxelles : De Boeck Université.

Van der Maren, J-M., Yvon, F. (2009). L'analyse du travail, entre parole et action. Recherches qualitatives. Hors série, 7 p 42-63.

Verret, M. (1975). Le temps des études, Librairie Honoré Champion. Paris ESF.

Vincent, P. (2007). Basket, méthode d'entrainement, la formation des joueurs. Paris: Chiron. 


\section{eJRIEPS 36 juillet 2015}

Vincent, P. (2007). Basket, la formation des joueurs. Paris : Chiron.

Yin, R. (1984). Case study research: Design and methods. Beverly Hills: Sage publications.

\section{Annexe 1}

\begin{tabular}{|c|c|c|}
\hline Catégories des savoirs & Types d'objets de savoirs & Abréviations \\
\hline $\begin{array}{l}\text { Les savoirs techniques } \\
(S T):\end{array}$ & $\begin{array}{l}\text { - Savoirs techniques sur le dribble } \\
\text { - Savoirs techniques sur la passe } \\
\text { - Savoirs technique sur le tir } \\
\text { - Savoirs techniques sur le rebond } \\
\text { - Savoirs techniques sur le démarquage } \\
\text { - Savoirs techniques sur la feinte } \\
\text { - Savoirs techniques sur la défense } \\
\text { - Savoirs techniques sur les appuis } \\
\text { - Savoirs techniques sur les écrans }\end{array}$ & $\begin{array}{l}\rightarrow \text { STD } \\
\rightarrow \text { STP } \\
\rightarrow \text { STT } \\
\rightarrow \text { STR } \\
\rightarrow \text { STDém } \\
\rightarrow \text { STF } \\
\rightarrow \text { STDéf } \\
\rightarrow \text { STApp } \\
\rightarrow \text { STE }\end{array}$ \\
\hline $\begin{array}{l}\text { Les savoirs tactiques } \\
\text { (STA): }\end{array}$ & $\begin{array}{l}\text { - La lecture du jeu } \\
\text { - La prise de décision } \\
\text { - La gestion tactique }\end{array}$ & $\begin{array}{l}\rightarrow \text { STAL } \\
\rightarrow \text { STAP } \\
\rightarrow \text { STAG }\end{array}$ \\
\hline $\begin{array}{l}\text { Les savoirs } \\
\text { méthodologiques } \\
(S M):\end{array}$ & $\begin{array}{l}\text { - La réglementation } \\
\text { - Le leadership } \\
\text { - La communication }\end{array}$ & $\begin{array}{l}\rightarrow \text { SMR } \\
\rightarrow \text { SML } \\
\rightarrow \text { SMC }\end{array}$ \\
\hline
\end{tabular}

\section{Annexe 2}




\begin{tabular}{|c|c|}
\hline Catégories de savoirs & $\begin{array}{c}\text { Pierre } \\
\text { 1ère séance }\end{array}$ \\
\hline Savoirs techniques (ST) & 130 \\
\hline STD & 19 \\
\hline STP & 26 \\
\hline STT & 34 \\
\hline STR & 0 \\
\hline STDém & 0 \\
\hline STF & 14 \\
\hline STDéf & 0 \\
\hline STApp & 37 \\
\hline Savoirs tactiques (STA) & 114 \\
\hline STAL & 23 \\
\hline STAP & 32 \\
\hline STAG & 59 \\
\hline Savoirs méthodologiques (SM) & 9 \\
\hline SMR & 5 \\
\hline SML & 0 \\
\hline SMC & 4 \\
\hline $\begin{array}{l}\text { Total objets de savoirs } \\
\text { réellement enseignés }\end{array}$ & 253 \\
\hline
\end{tabular}

\begin{tabular}{|c|c|}
\hline Catégories de savoirs & $\begin{array}{c}\text { Pierre } \\
2^{\text {ème }} \text { séance }\end{array}$ \\
\hline Savoirs techniques (ST) & 79 \\
\hline STD & 3 \\
\hline STP & 15 \\
\hline STT & 15 \\
\hline STR & 3 \\
\hline STDém & 0 \\
\hline STF & 4 \\
\hline STDéf & 26 \\
\hline STApp & 13 \\
\hline Savoirs tactiques (STA) & 18 \\
\hline STAL & 3 \\
\hline STAP & 4 \\
\hline STAG & 11 \\
\hline Savoirs méthodologiques (SM) & 8 \\
\hline SMR & 1 \\
\hline SML & 0 \\
\hline SMC & 7 \\
\hline $\begin{array}{l}\text { Total objets de savoirs } \\
\text { réellement enseignés }\end{array}$ & 105 \\
\hline
\end{tabular}

\begin{tabular}{|c|c|}
\hline Catégories de savoirs & $\begin{array}{c}\text { Pierre } \\
3^{\text {ème }} \text { séance }\end{array}$ \\
\hline Savoirs techniques (ST) & 61 \\
\hline STD & 15 \\
\hline STP & 10 \\
\hline STT & 0 \\
\hline STR & 4 \\
\hline STDém & 18 \\
\hline STF & 4 \\
\hline STDéf & 3 \\
\hline STApp & 7 \\
\hline Savoirs tactiques (STA) & 80 \\
\hline STAL & 31 \\
\hline STAP & 12 \\
\hline STAG & 37 \\
\hline Savoirs méthodologiques (SM) & 3 \\
\hline SMR & 1 \\
\hline SML & 1 \\
\hline SMC & 1 \\
\hline $\begin{array}{l}\text { Total objets de savoirs } \\
\text { réellement enseignés }\end{array}$ & 144 \\
\hline
\end{tabular}

\begin{tabular}{|c|c|}
\hline Catégories de savoirs & $\begin{array}{c}\text { Pierre } \\
\text { 4ème séance }\end{array}$ \\
\hline Savoirs techniques (ST) & 63 \\
\hline STD & 18 \\
\hline STP & 13 \\
\hline STT & 7 \\
\hline STR & 0 \\
\hline STDém & 6 \\
\hline STF & 3 \\
\hline STDéf & 10 \\
\hline STApp & 6 \\
\hline Savoirs tactiques (STA) & 44 \\
\hline STAL & 21 \\
\hline STAP & 1 \\
\hline STAG & 22 \\
\hline Savoirs méthodologiques (SM) & 6 \\
\hline SMR & 1 \\
\hline SML & 5 \\
\hline SMC & 0 \\
\hline $\begin{array}{l}\text { Total objets de savoirs } \\
\text { réellement enseignés }\end{array}$ & 113 \\
\hline
\end{tabular}

\section{Annexe 3}


eJRIEPS 36 juillet 2015

\begin{tabular}{|c|c|}
\hline Catégories de savoirs & $\begin{array}{c}\text { Pierre } \\
\text { 1ère séance }\end{array}$ \\
\hline Savoirs techniques (ST) & 6 \\
\hline STD & 0 \\
\hline STP & 1 \\
\hline STT & 5 \\
\hline STR & 0 \\
\hline STDém & 0 \\
\hline STF & 0 \\
\hline STDéf & 0 \\
\hline STApp & 0 \\
\hline STE & 0 \\
\hline Savoirs tactiques (STA) & 7 \\
\hline STAL & 0 \\
\hline STAP & 2 \\
\hline STAG & 5 \\
\hline Savoirs méthodologiques (SM) & $\mathbf{0}$ \\
\hline SMR & 0 \\
\hline SML & 0 \\
\hline SMC & 0 \\
\hline $\begin{array}{l}\text { Total des savoirs enseignés } \\
\text { théoriques }\end{array}$ & 13 \\
\hline
\end{tabular}

\begin{tabular}{|c|c|}
\hline Catégories de savoirs & $\begin{array}{c}\text { Pierre } \\
2^{\text {ème }} \text { séance }\end{array}$ \\
\hline Savoirs techniques (ST) & 17 \\
\hline STD & 0 \\
\hline STP & 1 \\
\hline STT & 2 \\
\hline STR & 0 \\
\hline STDém & 0 \\
\hline STF & 0 \\
\hline STDéf & 8 \\
\hline STApp & 3 \\
\hline STE & 3 \\
\hline Savoirs tactiques (STA) & 4 \\
\hline STAL & 0 \\
\hline STAP & 0 \\
\hline STAG & 4 \\
\hline Savoirs méthodologiques (SM) & 7 \\
\hline SMR & 1 \\
\hline SML & 0 \\
\hline SMC & 6 \\
\hline $\begin{array}{l}\text { Total des savoirs enseignés } \\
\text { théoriques }\end{array}$ & 28 \\
\hline
\end{tabular}

\begin{tabular}{|c|c|}
\hline Catégories de savoirs & $\begin{array}{c}\text { Pierre } \\
\text { 3ème }^{\text {séance }}\end{array}$ \\
\hline Savoirs techniques (ST) & 3 \\
\hline STD & 2 \\
\hline STP & 1 \\
\hline STT & 0 \\
\hline STR & 0 \\
\hline STDém & 0 \\
\hline STF & 0 \\
\hline STDéf & 0 \\
\hline STApp & 0 \\
\hline STE & 0 \\
\hline Savoirs tactiques (STA) & 14 \\
\hline STAL & 12 \\
\hline STAP & 1 \\
\hline STAG & 1 \\
\hline Savoirs méthodologiques (SM) & $\mathbf{0}$ \\
\hline SMR & 0 \\
\hline SML & 0 \\
\hline SMC & 0 \\
\hline $\begin{array}{l}\text { Total des savoirs enseignés } \\
\text { théoriques }\end{array}$ & 17 \\
\hline
\end{tabular}

\begin{tabular}{|c|c|}
\hline Catégories de savoirs & $\begin{array}{c}\text { Pierre } \\
\text { 4ème séance }\end{array}$ \\
\hline Savoirs techniques (ST) & 12 \\
\hline STD & 3 \\
\hline STP & 9 \\
\hline STT & 0 \\
\hline STR & 0 \\
\hline STDém & 0 \\
\hline STF & 0 \\
\hline STDéf & 0 \\
\hline STApp & 0 \\
\hline STE & 0 \\
\hline Savoirs tactiques (STA) & 7 \\
\hline STAL & 4 \\
\hline STAP & 2 \\
\hline STAG & 1 \\
\hline Savoirs méthodologiques (SM) & $\mathbf{0}$ \\
\hline SMR & 0 \\
\hline SML & 0 \\
\hline SMC & 0 \\
\hline $\begin{array}{l}\text { Total des savoirs enseignés } \\
\text { théoriques }\end{array}$ & 19 \\
\hline
\end{tabular}

